\title{
C3 Glomerulonephritis and Plasma Cell Dyscrasia: Expanding the Etiologic Spectrum
}

\author{
Dennis L Cooper ${ }^{1}$, William R Munday² and Gilbert W Moeckel ${ }^{2}$
}

Yale University School of Medicine, New Haven, CT, USA

\begin{abstract}
Background: C3 glomerulopathy (C3GP) including dense deposit disease (DDD) is mediated by abnormal activation of the alternative complement pathway (ACP). In children and young adults, mutations of complement or complement regulatory proteins are the major causative factors but in adults there appears to be an increased incidence of monoclonal gammopathy and it has been proposed that the paraprotein is functioning as a C3 nephritic factor or through other unknown mechanisms resulting in abnormal ACP activity.

We describe five patients with C3GP and plasma cell dyscrasias including two patients with symptomatic multiple myeloma and three patients with monoclonal gammopathy of renal significance one of whom progressed to symptomatic myeloma. One patient with DDD and elevated C3 nephritic factor responded to myeloma therapy with cyclophosphamide plus bortezomib and dexamethasone while another patient seemed to rapidly worsen both times after receiving lenalidomide, a drug with potent immunomodulatory activity. In two patients, the effect of myeloma therapy was indeterminate secondary to advanced disease. Two patients with renal transplant had recurrence C3GP in the transplanted kidney at 2 months and four years, respectively.
\end{abstract}

Conclusion: Adult patients with C3GP should be screened for plasma cell dyscrasias. Further studies are required to assess the value of myeloma-directed treatment and/or ACP inhibition.

Keywords: Monoclonal; Plasma cell; C3 Glomerulonephritis; C3 Convertase; Eculizemab

\section{Abbreviations}

C3GP: C3 Glomerulopathy; DDD: Dense Deposit Disease; ACP: Alternative Complement Pathway; MPGN: Membranoproliferative Glomerulonephritis; CRP: Complement Regulatory Proteins; MGRS: Monoclonal Gammopathy Of Renal Significance; Smac: Soluble Membrane Attack Complex

\section{Background}

Membranoproliferative glomerulonephritis (MPGN) is a descriptive term for abnormal glomerular changes characterized by distinct histological findings. Although originally categorized by the location of abnormal immune complex deposits on electron microscopy (EM), recently a more pathogenetic classification is used that relies on immunohistochemistry for the presence or absence of abnormal immunoglobulin and C3 [1,2]. The presence of significant immunoglobulin staining is associated with an immune complex etiology whereas dominant staining for C3 with little or no immunoglobulin is more indicative of abnormal activation of the alternative complement pathway (ACP). In the latter situation, the location and appearance of the deposits by EM can distinguish dense deposit disease (DDD, formerly type II MPGN) from other types of C3 glomerulonephritis (C3GP). Particularly, as C3GP and DDD may be amenable to emerging treatments that interrupt the ACP such as eculizumab [3] and other agents in development [4], the newer classification has potential therapeutic implications apart from the assignment of pathophysiology.

Monoclonal gammopathy has been associated with both immune complex type MPGN and C3GP, including DDD. The incidence of monoclonal gammopathy in patients with MPGN may be as high as $41 \%$ [5]. In patients with immune complex type MPGN, the paraprotein likely forms part of the immune complex causing activation of the classic complement pathway and resulting in renal injury with both abnormal immunoglobulin and complement on immunohistochemistry.

Similarly, while DDD [6,7] and C3GP [8] are much more common in children and young adults and are often associated with mutations in complement components or complement regulatory proteins (CRP), in adult patients with C3GP, $31 \%$ of patients were found to have a monoclonal gammopathy [9]. This number increased to $71 \%$ in adults with DDD [10]. Most of these patients did not have symptomatic multiple myeloma and would now be considered to have monoclonal gammopathy of renal significance (MGRS) [11]. With respect to pathophysiology of paraproteinemia-associated C3GP and DDD, it has been suspected but not proved that the paraprotein is functioning as an autoantibody that stabilizes $\mathrm{C} 3$ convertase (C3 nephritic factor) or possibly inhibits one of the CRPs, such as Factor H or I $[9,10]$. In this report, we confirm and expand the association of $\mathrm{C} 3 \mathrm{G}$ and plasma cell dyscrasias, including symptomatic multiple myeloma. We also show an apparent response to treatment with cyclophosphamide and bortezomib in a patient with DDD.

\section{Case Series}

We report five patients with plasma cell dyscrasia, four had C3GP and one had DDD proven by kidney biopsies. Clinical history and lab values

*Corresponding author: Gilbert W. Moeckel, MD, PhD, Department of Pathology 310 Cedar Street, LB20, PO Box 208023, New Haven, CT 06520-8023, USA, Tel: 203-737-2803; Fax: 203-785-3348; E-mail: gilbert.moeckel@yale.edu

Received August 13, 2015; Accepted September 30, 2015; Published October 07, 2015

Citation: Cooper DL, Munday WR, Moeckel GW (2015) C3 Glomerulonephritis and Plasma Cell Dyscrasia: Expanding the Etiologic Spectrum. Biol Med (Aligarh) 7: 252. doi: 10.4172/0974-8369.1000252

Copyright: (c) 2015 Cooper DL, et al. This is an open-access article distributed under the terms of the Creative Commons Attribution License, which permits unrestricted use, distribution, and reproduction in any medium, provided the original author and source are credited. 


\begin{tabular}{|c|c|c|c|c|c|c|c|}
\hline JPatent \# & Age/Sex & Serum $\mathrm{Cr}$ & SPEP/IFE & $\begin{array}{c}\text { Spot urine } P / C \\
(n \mid<0.1)\end{array}$ & $\mathrm{C} 3 / \mathrm{C} 4$ & $\begin{array}{c}\text { C3Nef } \\
\mathrm{nl}<0.3\end{array}$ & $\begin{array}{c}\text { sMAC } \\
\mathrm{nl}<244\end{array}$ \\
\hline 1 & $79 / F$ & 2.7 & $\begin{array}{l}1.5 \mathrm{gm} / \mathrm{dl} \\
\text { lgG-kappa }\end{array}$ & 0.7 & $\mathrm{NI} / \mathrm{NI}$ & NA & NA \\
\hline 2 & $67 / F$ & 7.2 & $\begin{array}{c}1.9 \mathrm{gm} / \mathrm{dl} \\
\operatorname{lgG}-\mathrm{kappa}\end{array}$ & NA & $\mathrm{L} / \mathrm{L}$ & 0.42 & H 606 \\
\hline 3 & $67 / \mathrm{M}$ & 4.4 & $\begin{array}{c}1.0 \mathrm{gm} / \mathrm{dl} \\
\lg \mathrm{G}-\mathrm{lambda}\end{array}$ & 5.2 & NA & 0.33 & H 460 \\
\hline 4 & $81 / F$ & 6.2 & $\begin{array}{c}1.0 \mathrm{gm} / \mathrm{dl} \\
\text { lgG/lgG lambda }\end{array}$ & 6.8 & $\mathrm{NI} / \mathrm{NI}$ & 0.66 & NA \\
\hline 5 & $57 / F$ & 1.4 & $\begin{array}{c}3.2 \mathrm{gm} / \mathrm{dl} \\
\operatorname{lgG} \text { lambda }\end{array}$ & 1.7 & NA & NA & H 1200 \\
\hline
\end{tabular}

Table 1: Patient demographics and initial laboratory results. Cr:creatinine; SPEP: serum protein electrophoresis; IFE: immunofixation electrophoresis; P/C: protein: creatinine ratio; C3Nef: C3 nephritic factor; sMAC: soluble membrane attack complex; NA: not assessed; NI: normal

are summarized in Table 1 . Patient 1 had a 2-yr history of hematuria, proteinuria and renal insufficiency and a bone marrow biopsy showed $15 \%$ clonal plasmacytosis. A renal biopsy showed MPGN and she was followed without treatment but eventually developed both ESRD and symptomatic myeloma with a large lytic bony lesion. She was treated with a modified cyclophosphamide, bortezomib, dexamethasone (CyBorD) regimen [12] and had a near complete serologic response. She has remained on hemodialysis.

Patient 2 was diagnosed with symptomatic multiple myeloma with lytic bony lesions and presented with acute renal failure two weeks after starting lenalidomide/dexamethasone. A renal biopsy showed ATN and C3GP. She had a partial serologic response and improvement in her renal function after treatment with modified CyBord but then had therapy changed to lenalidomide and dexamethasone secondary to worsening neuropathy. She quickly developed worsening renal insufficiency and hematuria. Lenalidomide was stopped and her renal function stabilized.

Patient 3 had a history of MPGN with gradually worsening renal function with a stable paraprotein and normal free light chain ratio. A review of a previous kidney biopsy established the diagnosis of C3GP. He was treated with modified CyBorD but did not show a serologic response. He developed ESRD and underwent renal transplantation. Because of increasing creatinine, hematuria and proteinuria, he underwent a renal biopsy two months after transplant that showed recurrent C3GP. He has been started on eculizumab.

Patient 4 presented with hematuria, renal failure and nephrotic range proteinuria. A renal biopsy showed DDD. Bone marrow biopsy showed 5\% clonal plasmacytosis. She did not respond to high dose steroids and was started on a modified CyBorD regimen. She achieved a partial serologic response and improvement in her renal function and proteinuria. She remains on bortezomib/dexamethasone maintenance nearly 18 months after the diagnosis of DDD.

Patient 5 was status post-renal transplant for MPGN. Although she had a diagnosis of smoldering multiple myeloma for 2-3 years, she did not receive treatment until being considered for a renal transplant. Prior to a living-related donor transplant, she was treated with bortezomib plus dexamethasone and achieved a serologic complete remission. About four years after kidney transplant, she had evidence of an increasing paraprotein and a bone marrow showed $20 \%$ plasmacytosis. She was then evaluated for recurrent hematuria and worsening renal function. A biopsy of the renal allograft showed C3GP and she was started back on modified CyBorD and continued tacrolimus immunoprophylaxis for the kidney transplant. She has not been followed long enough to assess her response.

\section{Discussion}

Our study confirms and expands previous series showing a relationship between C3GP (including DDD) and plasma cell dyscrasia $[9,10]$. In contrast to the latter reports that showed most patients had monoclonal gammopathy of undetermined significance (MGUS), one of our patients was diagnosed with C3GP just after the diagnosis of symptomatic MM and a second patient evolved from smoldering to symptomatic myeloma a few years after the diagnosis of MPGN (retrospectively reviewed and confirmed as C3GP). In addition, patient 5 developed recurrence of $\mathrm{C} 3 \mathrm{GP}$ in the setting of a rising paraprotein and $20 \%$ plasmacytosis. Thus far, only the patient with DDD has had a meaningful response to myeloma-based treatment (improved creatinine and decrease in proteinuria) but two others probably had disease that was too far advanced to show renal recovery.

The relationship between paraproteinemia and ACP dysregulation is unclear. However, patient 4 with DDD had a significantly increased C3 nephritic factor that normalized after treatment. Three patients $(2,3$ and 5) showed high levels of soluble membrane attack complex (sMAC) two of whom also had borderline high C3 nephritic factor levels. High sMAC is consistent with increased C5 convertase activity and may serve as a biomarker predicting favorable response to the $\mathrm{C} 5$ convertase inhibitor, eculizumab [3].

As it has been recognized that $\mathrm{C} 3 \mathrm{GP}$ and $\mathrm{DDD}$ are due to dysregulation of the ACP, there has been growing interest in the use of eculizumab, particularly given its effectiveness in treating paroxysmal nocturnal hemoglobinuria [13] and atypical hemolytic anemia [14,15], two other diseases mediated by abnormal control of ACP. Thus far, there have been few patients with C3GP that have been treated with eculizumab, recently summarized by Bomback [3]. In eight case reports, 7 of 8 patients responded [16-23]. In an open label series of six C3GP patients (3 patients had DDD), clinical and/or pathologic improvement was seen in 4 patients, but two patients experienced worsening disease. These findings indicate that eculizumab might not be appropriate for all patients with C3GP [24,25]. Importantly, the sMAC normalized in all patients who had elevations before eculizumab therapy and the two patients who had worsening renal function had normal sMAC levels before treatment (Figure 1).

A recent report has advocated treatment of the underlying disorder in patients with MGRS [26]. However, there have been very few reports of improvement in renal function/proteinuria following treatment. In patients with symptomatic and smoldering multiple myeloma it is certainly reasonable to treat the underlying plasma cell dyscrasia. However, it should be noted that complications such as MGUS-associated neuropathy inconsistently respond to treatment of the underlying disorder [27]. Our patient 2 had dramatic worsening of her renal function on two occasions shortly after treatment with 

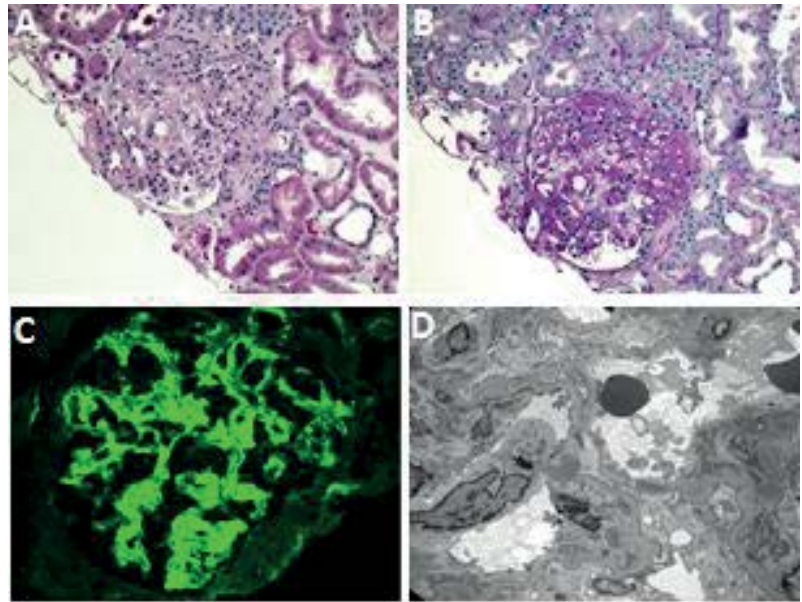

Figure 1: Representativelight, immnofluorescence, and electronmicroscopy in patient 3 with $\mathrm{C} 3$ glomerulopathy and monoclonal gammopathy. (A, B) Light microscopy demonstrates a membranoproliferative pattern of injury. [A] Hematoxylin \& Eosin stain, 20X; [B] Periodic acid-Schiff stain, 20X (C) Immunofluorescence shows bright $\mathrm{C} 3$ in the mesangium and capillary loops, 400x. (D) Electron microscopy shows numerous subendothelial deposits and occasional subepithelial deposits.

the immunomodulatory drug (IMiD) lenalidomide suggesting that activation of the immune system by an IMiD may have worsened her disease $[28,29]$.

It seems likely that C3GP is under-diagnosed. Indeed, three of the five patients in the current series were initially diagnosed as MPGN. The diagnosis of C3GP was only established after either repeat biopsies or review of older ones. Since awareness of the diagnostic criteria has recently increased, there will likely be an increase in patients diagnosed with C3GP.

\section{Conclusion}

Our study strengthens the evidence that paraproteinemia is an underlying etiology of C3GP. In the future it will be important to determine whether patients like these should receive treatment for symptomatic MM, complement inhibitors alone or both.

\section{Competing Interests}

The authors declare that they have no competing interests

\section{Authors Contribution}

DC and GM conceived of the study, WM and DC compiled the clinical data, all authors have read the manuscript.

\section{Consent}

"Written informed consent was obtained from the patient for publication of this case report and any accompanying images. A copy of the written consent is available for review by the Editor of this journal."

\section{References}

1. Sethi S, Fervenza FC (2012) Membranoproliferative glomerulonephritis--a new look at an old entity. N Engl J Med 366: 1119-1131.

2. Sethi S, Nester CM, Smith RJ (2012) Membranoproliferative glomerulonephritis and C3 glomerulopathy: resolving the confusion. Kidney Int 81: 434-441.

3. Bomback AS (2014) Eculizumab in the Treatment of Membranoproliferative
Glomerulonephritis. Nephron Clin Pract. 128:270-276.

4. Qu H, Ricklin D, Bai H, Chen H, Reis ES, et al. (2013) New analogs of the clinical complement inhibitor compstatin with subnanomolar affinity and enhanced pharmacokinetic properties. Immunobiology 218: 496-505.

5. Sethi S, Zand L, Leung N, Smith RJ, Jevremonic D, et al. (2010) Membranoproliferative glomerulonephritis secondary to monoclonal gammopathy. Clin J Am Soc Nephrol 5: 770-782.

6. Lu DF, Moon M, Lanning LD, McCarthy AM, Smith RJ (2012) Clinical features and outcomes of 98 children and adults with dense deposit disease. Pediatr Nephrol 27: 773-781.

7. Nasr SH, Valeri AM, Appel GB, Sherwinter J, Stokes MB, et al. (2009) Dense deposit disease: clinicopathologic study of 32 pediatric and adult patients. Clin J Am Soc Nephrol 4: 22-32.

8. Servais A, Noel LH, Roumenina LT, Le Quintrec M, Ngo S, et al. (2012) Acquired and genetic complement abnormalities play a critical role in dense deposit disease and other C3 glomerulopathies. Kidney Int 82: 454-464.

9. Zand L, Kattah A, Fervenza FC, Smith RJ, Nasr SH, et al. (2013) C3 glomerulonephritis associated with monoclonal gammopathy: a case series. Am J Kidney Dis 62: 506-514.

10. Sethi S, Sukov WR, Zhang Y, Fervenza FC, Lager DJ, et al. (2010) Dense deposit disease associated with monoclonal gammopathy of undetermined significance. Am J Kidney Dis 56: 977-982.

11. Leung N, Bridoux F, Hutchison CA, Nasr SH, Cockwell P, et al. (2012) Monoclonal gammopathy of renal significance: when MGUS is no longer undetermined or insignificant. Blood 120: 4292-4295.

12. Mikhael JR, Schuster SR, Jimenez-Zepeda VH, Bello N, Spong J, et al. (2012) Cyclophosphamide-bortezomib-dexamethasone (CyBorD) produces rapid and complete hematologic response in patients with $\mathrm{AL}$ amyloidosis. Blood 119 4391-4394.

13. Hillmen P, Young NS, Schubert J, Brodsky RA, Socie G, et al. (2006) The complement inhibitor eculizumab in paroxysmal nocturnal hemoglobinuria. $\mathrm{N}$ Engl J Med 355: 1233-1243.

14. Gruppo RA, Rother RP (2009) Eculizumab for congenital atypical hemolyticuremic syndrome. N Engl J Med 360: 544-546.

15. Nurnberger J, Philipp T, Witzke O, Opazo Saez A, Vester U, et al. (2009) Eculizumab for atypical hemolytic-uremic syndrome. N Engl J Med 360: 542544

16. Daina E, Noris M, Remuzzi G (2012) Eculizumab in a patient with densedeposit disease. N Engl J Med 366: 1161-1163.

17. Gurkan S, Fyfe B, Weiss L, Xiao X, Zhang Y, et al. (2013) Eculizumab and recurrent C3 glomerulonephritis. Pediatr Nephrol 28: 1975-1981.

18. Kerns E, Rozansky D, Troxell ML (2013) Evolution of immunoglobulin deposition in C3-dominant membranoproliferative glomerulopathy. Pediatr Nephrol 28 2227-2231.

19. McCaughan JA, O'Rourke DM, Courtney AE (2012) Recurrent dense deposit disease after renal transplantation: an emerging role for complementary therapies. Am J Transplant 12: 1046-1051.

20. Ozkaya O, Nalcacioglu H, Tekcan D, Genc G, Meydan BC, et al. (2014) Eculizumab therapy in a patient with dense-deposit disease associated with partial lipodystropy. Pediatr Nephrol 29: 1283-1287.

21. Radhakrishnan S, Lunn A, Kirschfink M, Thorner P, Hebert D, et al. (2012) Eculizumab and refractory membranoproliferative glomerulonephritis. N Eng J Med 366: 1165-1166.

22. Rousset-Rouviere C, Cailliez M, Garaix F, Bruno D, Laurent D, et al. (2014) Rituximab fails where eculizumab restores renal function in C3nef-related DDD. Pediatr Nephrol 29: 1107-1111.

23. Vivarelli $M$, Pasini A, Emma $F$ (2012) Eculizumab for the treatment of densedeposit disease. N Engl J Med 366: 1163-1165.

24. BombackAS, Smith RJ, Barile GR, Zhang Y, Heher EC, et al. (2012) Eculizumab for dense deposit disease and C3 glomerulonephritis. Clin J Am Soc Nephrol 7: 748-756.

25. Herlitz LC, Bomback AS, Markowitz GS, Stokes MB, Smith RN, et al. (2012) Pathology after eculizumab in dense deposit disease and C3 GN. J Am Soc 
Citation: Cooper DL, Munday WR, Moeckel GW (2015) C3 Glomerulonephritis and Plasma Cell Dyscrasia: Expanding the Etiologic Spectrum. Biol Med (Aligarh) 7: 252. doi: 10.4172/0974-8369.1000252

Page 4 of 4

Nephrol 23: 1229-1237.

26. Sethi S, Rajkumar SV (2013) Monoclonal gammopathy-associated proliferative glomerulonephritis. Mayo Clin Proc 88: 1284-1293.

27. Ramchandren S, Lewis RA (2012) An update on monoclonal gammopathy and neuropathy. Curr Neurol Neurosci Rep 12: 102-110.
28. Brauer DL, Edelman B, Rapoport AP, Hess JR, Akpek G (2012) Plasma exchange and rituximab treatment for lenalidomide-associated cold agglutinin disease. Transfusion 52: 2432-2435.

29. Darabi K, Kantamnei S, Wiernik PH (2006) Lenalidomide-induced warm autoimmune hemolytic anemia. J Clin Oncol 24: e59. 